\title{
Efficacy and safety of aflibercept in metastatic colorectal cancer pretreated with bevacizumab: A report of five cases
}

\author{
JULIA ALCAIDE ${ }^{1}$, MAYTE DELGADO ${ }^{2}$, MARTA LEGERÉN ${ }^{2}$, JOSÉ MIGUEL JURADO ${ }^{2}$, \\ ISABEL BLANCAS ${ }^{2}$, TERESA PEREDA ${ }^{3}$, JORGE LÓPEZ ${ }^{3}$, MARGARITA GARRIDO ${ }^{4}$, \\ MARÍA J. SÁNCHEZ ${ }^{2}$, JOSÉ L. GARCÍA ${ }^{2}$ and ANTONIO RUEDA ${ }^{1}$ \\ ${ }^{1}$ Oncology Department, Costa del Sol Hospital, Marbella, 29603 Málaga; \\ ${ }^{2}$ Oncology Department, San Cecilio Clinical Hospital, Granada, 18014 Andalucía; ${ }^{3}$ Pathology Department \\ and ${ }^{4}$ Pharmacy and Nutrition Department, Costa del Sol Hospital, Marbella, 29603 Málaga, Spain
}

Received April 8, 2015; Accepted June 27, 2016

DOI: $10.3892 / \mathrm{ol} .2016 .5068$

\begin{abstract}
Aflibercept is a recombinant fusion protein that acts by inhibiting tumoural angiogenesis. Efficacy data obtained in the VELOUR randomised study has contributed to the approval of aflibercept as a second-line metastatic colorectal cancer (mCRC) treatment following an oxaliplatin-based regimen. The present study reports a case series of five patients with mCRC, who were treated in two centres since 2011 in the Compassionate Use Program for aflibercept. All patients had a KRAS mutation and previously received palliative fluoropyrimidine-oxaliplatin-based chemotherapy with bevacizumab. A doublet with irinotecan combined with aflibercept was administered until progression of disease. The majority of patients received a greater number of aflibercept cycles than the median reported in the VELOUR study (12 vs. 7 cycles), with manageable and reversible toxicity. The most frequent adverse events observed were diarrhoea, neutropenia, fatigue, proteinuria and hypertension. Most cases obtained a progression-free survival greater than the median reported in the VELOUR study (11 vs. 6.9 months) and, in a subgroup of patients previously treated with bevacizumab, and a median survival time of $\sim 47$ months was reached from the initial treatment of the disease. The present study contrasts the efficacy and safety results obtained from the pivotal VELOUR trial, and confirms that aflibercept, used in routine clinical practice outside of the clinical trial environment, is active and well-tolerated following bevacizumab treatment.
\end{abstract}

Correspondence to: Dr Mayte Delgado, Oncology Department, San Cecilio Clinical Hospital, Dr Oloriz Avenue, Granada, 18014 Andalucía, Spain

E-mail: maitechu22@hotmail.com

Abbreviations: mCRC, metastatic colorectal cancer; VEGF, anti-vascular endothelial growth factor; ECOG, eastern cooperative oncology Group; PS, performance status; CT, computed tomography; TRG, tumour regression grade; EGFR, epidermal growth factor receptor; PFS, progression-free survival

Key words: metastatic colorectal cancer, aflibercept, angiogenesis, targeted therapy, VEGF, VEGFR

\section{Introduction}

Worldwide, $\sim 1.2$ million patients are diagnosed with colorectal cancer each year (1). Up to one-quarter of these patients present with advanced disease at diagnosis, and $\sim 25 \%$ of patients with early stage disease relapse at some point during the disease course (2). Although prognosis has greatly improved over the past decades, due to significant surgical and medical advances, once the tumour has progressed beyond surgical resectability the disease is essentially incurable and the median survival is $\sim 2$ years with the best available systemic therapy (3). Treatment selection for patients is affected by the treatment objective, overall condition and comorbidity of the patient, and mutational state of the $R A S$ gene (4). It has recently been identified that patients that are not only selected by mutation status of the KRAS gene, but also the NRAS gene, improve when a drug targeting the epidermal growth factor receptor (EGFR) is incorporated into the treatment plan $(5,6)$. However, patients that present with a mutation in one of these genes may experience a detrimental effect on survival by combining an anti-EGFR drug with a chemotherapy regimen (5). Therefore, in patients with wild-type $R A S$ tumours (absence of mutations in exons 2, 3 and 4 of the KRAS gene, and in mutations in exons 2, 3 and 4 of the NRAS gene), it is currently possible to combine chemotherapy, including fluoropyrimidine, oxaliplatin and irinotecan-based therapies, with anti-EGFR therapies, including cetuximab and panitumumab, or with antiangiogenic agents, such as bevacizumab and aflibercept (2). In addition to their predictive value, $K R A S$ mutations have prognostic significance, since patients with mutated $K R A S$ appear to have lower survival rates compared with patients with wild-type KRAS (34.9 vs. 37.8 months, respectively) (7). Survival is even lower (21.2 months) in cases of metastatic colorectal cancer (mCRC), where mutations are expressed in the $B R A F$ gene (7). In addition to a poorer prognosis, this means that cases of mCRC with mutated RAS (KRAS and NRAS), which is $~ 50 \%$ of the $\mathrm{mCRC}$ population, have fewer treatment options available (6). In this group of patients the only biological agents that have demonstrated efficacy with chemotherapy are anti-vascular endothelial growth factor (VEGF) drugs, including bevacizumab and aflibercept. 
Bevacizumab is a humanised monoclonal antibody that binds to VEGF-A and prevents interaction between VEGF-A and VEGF receptor (VEGFR) (8,9). Bevacizumab is the only anti-VEGF agent approved for use in first-line treatment of $\mathrm{mCRC}$ and its efficacy is independent of $R A S$ family mutation status (10). By contrast, aflibercept is a genetically engineered fusion protein that contains VEGFR-2 receptor domain 1 and VEGFR-1 receptor domain 2, with the ability to 'sequester' all isoforms of VEGF, including VEGF-A, VEGF-B and placental growth factor (PIGF) (11). Aflibercept blocks the main VEGF factors that are involved in tumoural angiogenesis, unlike other antiangiogenic drugs that act against a single factor of the VEGF family (e.g. bevacizumab and VEGF-A). This allows the control of escape routes that are activated when only one of these factors is blocked. For example, increased levels of PIGF have been associated with resistance to bevacizumab (12). Aflibercept is approved in combination with irinotecan, 5-fluorouracil (5FU) and folinic acid treatment (FOLFIRI) in patients with $\mathrm{mCRC}$, who have disease progression following oxaliplatin treatment (13). Additionally, it is approved for use independent of the patient's $R A S$ gene family mutation status, Eastern Cooperative Oncology Group (ECOG) performance status (PS) or whether the patient has received bevacizumab (14). Subsequent to the granting of the Marketing Authorization in Europe (February 2012), a Compassionate Use Program was approved, which provided early access to aflibercept until it was marketed in Spain (September 2013),thus increasing the amount of safety data on the general population outside of randomised clinical trials.

The aim of the present study is to report the experience of patients that have been prescribed with aflibercept within the Compassionate Use Program at two centres: San Cecilio Clinical Hospital (Granada, Spain) and Costa del Sol Hospital (Marbella, Spain).

\section{Case report}

General patient information. All patients were characterised as having previously received one line of treatment, including another antiangiogenic agent, as follows: Bevacizumab combined with modified FOLFOX6 (FOLFOX6m) (oxaliplatin, $85 \mathrm{mg} / \mathrm{m}^{2}$ on day 1 ; leucovorin, $200 \mathrm{mg} / \mathrm{m}^{2}$ on day 1 ; $5 \mathrm{FU}$ bolus, $400 \mathrm{mg} / \mathrm{m}^{2}$ on day 1 ; $5 \mathrm{FU}$ continuous infusion, $2,400 \mathrm{mg} / \mathrm{m}^{2}$ on day 1 ; bevacizumab, $5 \mathrm{mg} / \mathrm{kg}$ on day $1 ; 14$ day cycle), or XELOX (capecitabine, $1,000 \mathrm{mg} / \mathrm{m}^{2}$ every $12 \mathrm{~h}$ on days $1-14$; oxaliplatin, $130 \mathrm{mg} / \mathrm{m}^{2}$ on day 1 ; bevacizumab $7.5 \mathrm{mg} / \mathrm{kg}$ on day $1 ; 21$ day cycle). At the progression of disease, the patients received FOLFIRI in combination with aflibercept (irinotecan, $180 \mathrm{mg} / \mathrm{m}^{2}$ on day 1; leucovorin, $200 \mathrm{mg} / \mathrm{m}^{2}$ on day 1 ; $5 \mathrm{FU}$ bolus, $400 \mathrm{mg} / \mathrm{m}^{2}$ on day 1 ; $5 \mathrm{FU}$ continuous infusion, 2,400 mg/m² on day 1 ; aflibercept, $4 \mathrm{mg} / \mathrm{kg}$ on day 1 ; 14 day cycle) for a specific number of cycles, which in many cases was greater than the median number of cycles received in the VELOUR study (11).

Table I summarizes the main clinical characteristics of the patients included in the present study, as well as the efficacy of the treatment administered in each of the cases. All patients presented with mCRC, as well as mutated KRAS in codon 12 , except for case 1 and 5, whose mutations were localised in codon 13 , specifically a G13D/13ASP mutation. Patients were informed about their inclusion in the compassionate use program by their treating physician and all gave their informed consent in writing prior to inclusion.

Case 1. A 39 year-old male presented to San Cecilio Clinical Hospital in October 2011 with rectal bleeding and epigastric pain, which had been ongoing for 4 months, with no other notable history. A colonoscopy was performed in October 2011, which revealed the presence of a stenosing mass $10-12 \mathrm{~cm}$ from the anal margin. A biopsy confirmed adenocarcinoma. Analysis revealed that the patient had a significant elevation in carcinoembryonic antigen (CEA; $140 \mathrm{ng} / \mathrm{ml}$; normal range, $0-5 \mathrm{ng} / \mathrm{ml}$ ) and carbohydrate antigen 19-9 (CA 19-9; $544 \mathrm{U} / \mathrm{ml}$; normal range, 0-37 U/ml). Other parameters were within the normal ranges. A thoracic/abdominal computerised tomography (CT) scan revealed the presence of three hepatic lesions (segments VII-VIII, $38 \mathrm{~mm}$ in size; segment II, $30 \mathrm{~mm}$; segments I-VI, $15 \mathrm{~mm}$ ), all of which were consistent with metastases. In the primary tumour biopsy, a KRAS mutation was identified at codon 13 of exon 2 (G13D or 13ASP). The patient received first-line chemotherapy with FOLFOX6m and bevacizumab, with markers normalising from the second cycle onward. Subsequent to 9 cycles, a partial radiological response was achieved, allowing intervention in May 2012 by lower anterior resection, bisegmentectomy II-III and metastasectomy of atypical segment VIII; no lesion was identified in segment I. The pathological stage of the tumour was pT3N0M1, with moderate differentiation and Mandard tumour regression grade (TRG) of 3, which indicates predominant fibrosis over residual cancerous tissue, with two adenocarcinoma liver metastases of $15 \mathrm{~mm}$ and $25 \mathrm{~mm}$. The patient completed 12 cycles of FOLFOX6m-bevacizumab, without oxaliplatin due to neurotoxicity, and received pelvic radiotherapy (45 Gy; $1.8 \mathrm{~Gy} /$ fraction). The patient underwent periodic review (tumour markers and CT evaluation every 3 months) with no evidence of disease progression in October 2012.

In February 2013, a CT scan revealed bilateral sub-centimetre pulmonary metastases and a $15 \mathrm{~mm}$ liver metastasis on segment I (Fig. 1). The Multidisciplinary Committee considered the patient a candidate for palliative chemotherapy. The patient had an ECOG PS score of 0, and received second line treatment with FOLFIRI-aflibercept, within the aflibercept Compassionate Use Program. A follow-up CT scan was performed in May 2013, following 7 cycles, which revealed a small remaining pulmonary nodule in the right upper lobe, another part of a nodule in the right lower lobe next to the sulcus, and another small nodule in the left lower lobe without hepatic involvement. The response assessment was partial response (PR) for the lungs and complete response (CR) for liver metastases. In August 2013, the patient completed 12 cycles of FOLFIRI-aflibercept with a complete radiological response (Fig. 2). Subsequently, the patient underwent periodic review. Tumour markers remained normal throughout the follow-up period.

In October 2013, the patient presented with a pulmonary relapse of three nodules in the same location. Thoracic surgery was ruled out due to multiple bilateral and subcentimetric metastases, as well as a short progression-free interval, and treatment was resumed with FOLFIRI-aflibercept. The patient received 14 additional cycles to date, with good tolerance and 
Table I. Characteristics of five patients included in the present study and their treatment response.

\begin{tabular}{|c|c|c|c|c|c|}
\hline Case no. & 1 & 2 & 3 & 4 & 5 \\
\hline Age, years & 40 & 54 & 66 & 57 & 60 \\
\hline Gender & Male & Female & Female & Female & Male \\
\hline PS, second line & 0 & 0 & 1 & 0 & 1 \\
\hline$K R A S$ mutation & $\begin{array}{c}\text { C } 13 \\
\text { (13Asp/G13D) }\end{array}$ & $\begin{array}{c}\text { C } 12 \\
(12 \mathrm{Asp} / \mathrm{G} 12 \mathrm{D})\end{array}$ & $\begin{array}{c}\mathrm{C} 12 \\
(12 \mathrm{Arg} / \mathrm{G} 12 \mathrm{R})\end{array}$ & $\begin{array}{c}\text { C 12 } \\
(12 \mathrm{Val} / \mathrm{G} 12 \mathrm{~V})\end{array}$ & $\begin{array}{c}\text { C } 13 \\
\text { (13Asp/G13D) }\end{array}$ \\
\hline Primary tumour location & Rectum & Rectum & Transverse colon & $\begin{array}{l}\text { Splenic angle } \\
\text { of the colon }\end{array}$ & $\begin{array}{l}\text { Hepatic angle } \\
\text { of the colon }\end{array}$ \\
\hline Metastasis location & Liver and lung & Liver and lung & Liver & $\begin{array}{l}\text { Liver and lymph } \\
\text { node }\end{array}$ & $\begin{array}{l}\text { Liver, lymph node } \\
\text { lung and bone }\end{array}$ \\
\hline No. of cycles, second line & 12 & 29 & 14 & 19 & 8 \\
\hline No. of cycles, aflibercept & $12^{\mathrm{a}}$ & 28 & $11^{\mathrm{a}}$ & 17 & 3 \\
\hline Radiological response & $\mathrm{CR}$ & SD & SD & PR & SD \\
\hline PFS, second line, months & 8 & 19 & 27 & 11 & 5 \\
\hline OS, second line, months & $>41$ & 36 & $>36$ & 17 & 6 \\
\hline OS, first line, months & $>56$ & 47 & $>67$ & 41 & 12 \\
\hline
\end{tabular}

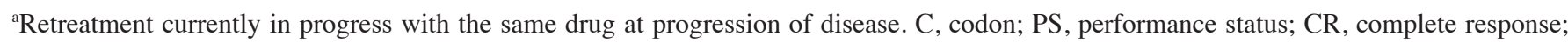
PR, partial response; SD, stabilisation of disease; PFS, progression-free survival; OS, overall survival.
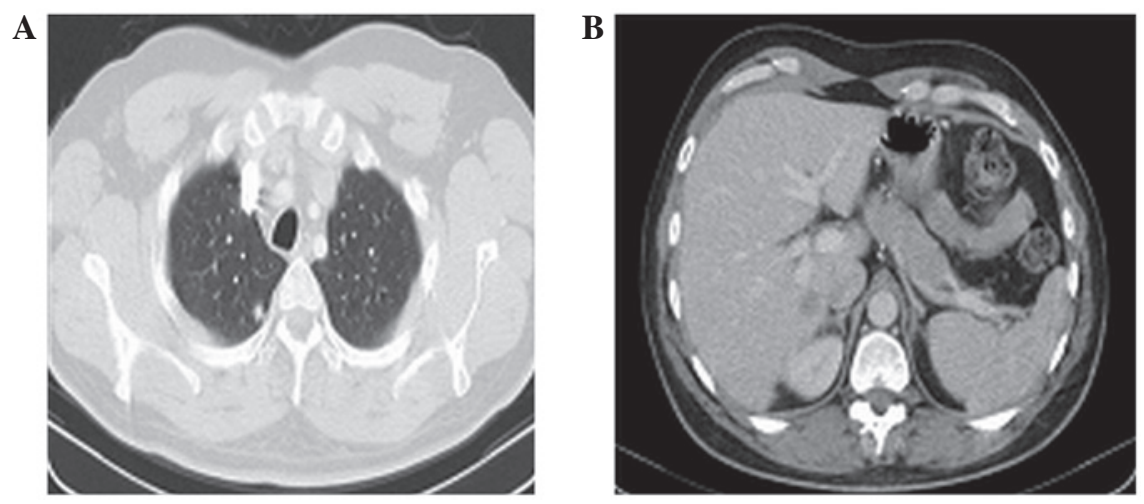

Figure 1. Case 1. Thoracic-abdominal computed tomography scan prior to administration of FOLFIRI (irinotecan, leucovorin and 5-fluorouracil)-aflibercept. (A) Pulmonary nodules ( $8 \mathrm{~mm}$ in size) at the apical segment of the right upper lobe and the left upper lobe. (B) A $15 \mathrm{~mm}$ hepatic lesion at segments I-VI consistent with metastasis.

radiologically stable disease. Toxicity during treatment was low, with grade 1-2 diarrhoea, and grade 1 alopecia and hypertension, which was controlled with enalapril and amlodipine. In cycles 5, 13 and 14, grade 2 proteinuria was detected, which prompted the administration of FOLFIRI without aflibercept. The patient is currently alive with pulmonary and hepatic disease, under regorafenib treatment.

Case 2. A 53 year-old woman presented to San Cecilio Clinical Hospital in February 2012 with increased intestinal transit and hematochezia. Following a positive faecal occult blood test, a colonoscopy revealed the presence of a mass $5-8 \mathrm{~cm}$ from the anal margin, which occupied the circumference of the lumen, but allowed endoscope entry. A biopsy confirmed the presence of invasive adenocarcinoma. Analysis revealed that the patient had increased levels of CEA $(269.5 \mathrm{ng} / \mathrm{ml})$ and CA 19-9 (1,096.0 U/ml). There was also evidence of multiple lung and liver metastases following a CT scan. Subsequent to detection of mutated KRAS in codon 12 of exon 2 (12Asp/G12D), the patient was administered with chemotherapy of FOLFOX6m-bevacizumab. Following 12 cycles, the patient continued chemotherapy with 5 additional cycles of 5FU/leucovorin-bevacizumab, without oxaliplatin, due to a hypersensitivity reaction. At follow-up in February 2013, the patient presented with tumour progression (Fig. 3), and second-line treatment with FOLFIRI-aflibercept was recommended. The patient had a PS score of 0 when second-line treatment was commenced. The patient received 29 cycles of FOLFIRI-aflibercept, with radiological disease stability (Fig. 4). Regarding toxicity, the patient presented with grade 2 palmar-plantar erythrodysesthesia, and recovered following a week of rest and a $15 \%$ reduction in the $5 \mathrm{FU}$ dose. Re-evaluation visits were made every 3 months to assess tumour markers and CT scans, until September 2014, when tumour progression was observed. The patient succumbed to the disease in February 2016. 

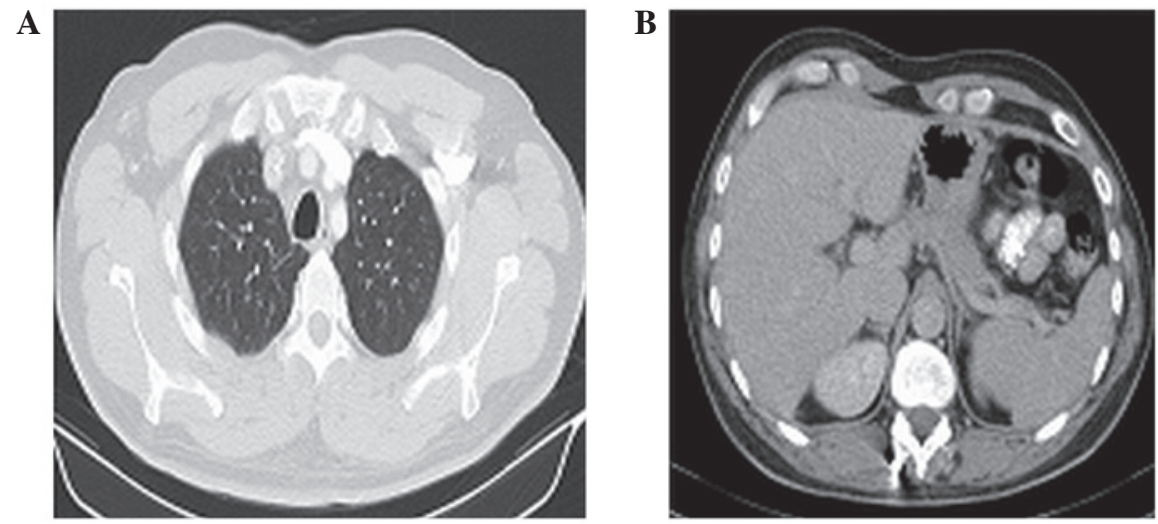

Figure 2. Case 1. Thoracic-abdominal computed tomography scan following 12 cycles of treatment with FOLFIRI (irinotecan, leucovorin and 5-fluorouracil)-aflibercept revealing a complete (A) pulmonary and (B) hepatic response.
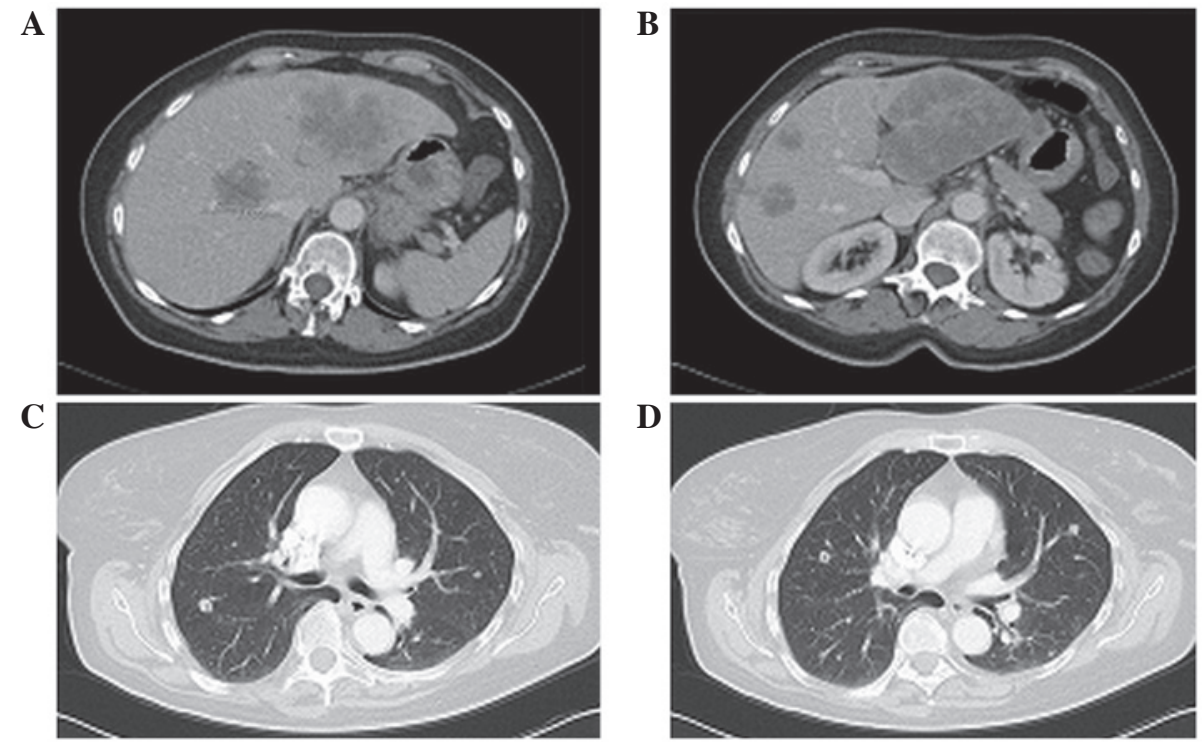

Figure 3. Case 2. Thoracic-abdominal computed tomography scan following FOLFOX6m (oxaliplatin, leucovorin and 5-fluorouracil)-bevacizumab treatment revealing tumour progression. (A) In September 2012, scans revealed hepatic lesions at segments IV $(4.5 \mathrm{~cm}$ in size), LI $(10 \mathrm{~cm}), \mathrm{VI}(2 \mathrm{~cm}), \mathrm{VIII}(2.2 \mathrm{~cm})$ and VIII (3.3 cm). (B) In February 2013, hepatic lesions were observed at segments IV (4.5 cm in size), LI (10 cm), VI (2 cm), VIII $(2.2 \mathrm{~cm})$ and VIII (3.3 cm). (C) In September 2012, right lung metastasis was detected. (D) In February 2013, bilateral pulmonary metastasis was detected.

Case 3. A 66 year-old woman presented to Costa del Sol Hospital in October 2010 with a history of hypertension and hypercholesterolemia, and was diagnosed with stage IV colon adenocarcinoma, due to liver metastases. A mutation at codon 12 of the KRAS gene (Arg12/G12R) was identified. Tumour markers (CEA and CA 19-9) were normal at baseline. In December 2010, the patient began treatment with FOLFOX6m in combination with bevacizumab for a total of 5 cycles. A follow-up CT scan (February 2011) revealed a slight reduction in liver metastases, which were more hypodense than before, hence a radiological stabilisation was noted based on Response Evaluation Criteria In Solid Tumours (15). The patient underwent surgical intervention in April 2011, with transverse colectomy, segmentectomy of VII and liver metastasectomy. The pathological stage of the tumour was pT3N1M1, and four liver metastases 1.7, 1.4, 0.8 and $5 \mathrm{~cm}$ in size were resected. The tumour had a Mandard TRG of 3. Three of the metastases extended focally to the margin of resection. Subsequent to surgery, the patient resumed treatment with FOLFOX-bevacizumab, and completed a total of 12 cycles of treatment in September 2011.
The patient was followed up with CEA and CT scans every 3-6 months until June 2013, when a single 15-mm liver recurrence was detected on a CT scan (Fig. 5A). The patient's tumour markers were normal and the patient had an ECOG PS of 1. Based on the localisation of the hepatic recurrence, it was not considered resectable or a candidate for ablation therapy. Treatment was initiated with aflibercept in combination with FOLFIRI within the Compassionate Use Program from July 2013, with an initial 75\% reduction in FOLFIRI dose, due to toxicity from a prior regimen. A CT scan subsequent to 6 cycles revealed the presence of a lesion $19 \mathrm{~mm}$ in diameter, with more defined contours, increased hypodensity, and without contrast enhancement, which was characterised as complete necrosis (Fig. 5B). The patient received a total of 14 cycles of treatment, 11 of these with aflibercept.

A CT scan in February 2014 did not reveal remission of the tumour. Therefore, following discussion of the case with the Multidisciplinary Committee, the patient underwent exploratory surgery and assessment of resection of liver metastasis in segment VIII in March 2014. Due to its anatomical location, 
A

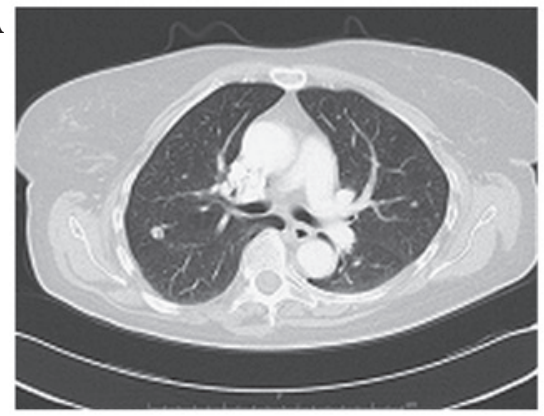

B

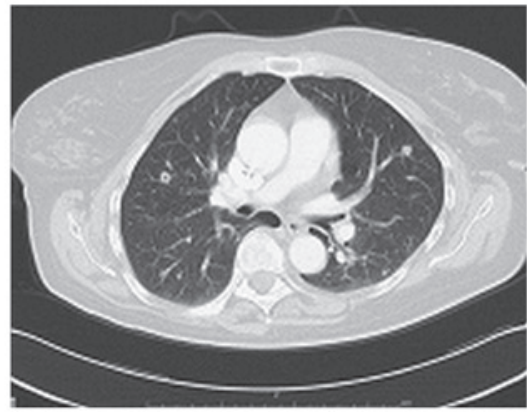

C

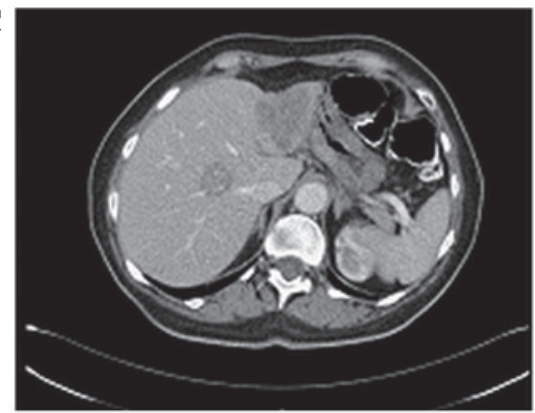

Figure 4. Case 2. Thoracic-abdominal computed tomography scan following FOLFIRI (irinotecan, leucovorin and 5-fluorouracil)-aflibercept treatment. (A and B) Multiple millimetric bilateral pulmonary nodules (right lower lobe, $8 \mathrm{~mm}$ in size; left lower lobe, $9 \mathrm{~mm}$ ) observed in (A) February 2013 and (B) April 2014, which were consistent with metastases. (C) Multiple metastatic hepatic nodules at segment II (53 mm) and segment VIII (21 mm).
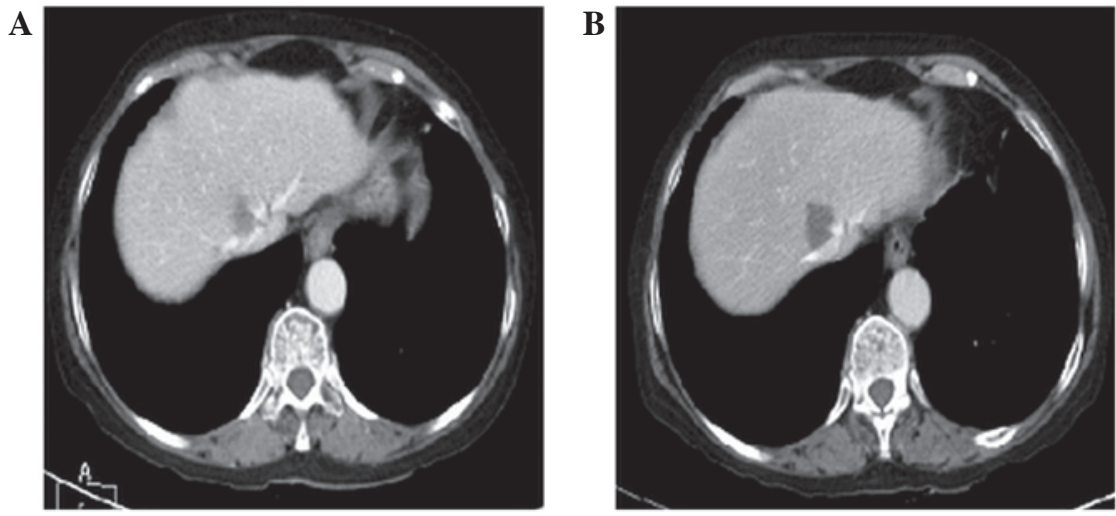

Figure 5. Case 3. Computed tomography scan at (A) baseline and (B) following 6 cycles of treatment with FOLFIRI (irinotecan, leucovorin and 5 -fluorouracil)-aflibercept. The scans revealed the presence of a hepatic lesion, which exhbited morphological response criteria; the lesion became more hypodense, homogeneous and well-defined, and did not present contrast uptake following treatment.

intraoperative ablation of the lesion was assessed prior to performing a resection to establish the surgical margins. However, on further inspection a second suspicious recurrent lesion was detected on the scar from the prior liver metastasectomy, which was attached to the diaphragm, making it unresectable. A biopsy of this lesion confirmed it as metastatic. Therefore, the surgical plan could not be completed. As predicted by the optimal morphological response obtained at imaging, the pathology report of the sample confirmed a significant Mandard TRG of 2, which indicates rare residual cancer cells. She experienced a new progression of the disease in October 2015, and was rechallenged with FOLFIRI-aflibercept. To date, the patient has achieved a survival of 67 months from the beginning of first-line treatment, and 36 months following second-line FOLFIRI-aflibercept-based treatment. Toxicity of the FOLFIRI treatment was moderate, with grade 1 diarrhoea, dysphonia, epistaxis and conjunctival toxicity, grade 2 alopecia, fatigue and hyporexia, and grade 3 neutropenia, which required a delay of cycle 12 . Blood pressure was controlled throughout treatment with the usual medication, and proteinuria was detected during the last cycle via urine test strip, with a urine protein-creatinine ratio of $<1$.

Case 4. A 57 year-old woman was diagnosed at Costa del Sol Hospital in May 2010 with stage IV adenocarcinoma of the colon, based on the identification of multiple liver metastases on a CT scan. In addition to a lesion in segment II, 8 lesions on the right hepatic lobe and a $12 \mathrm{~mm}$ adenopathy in the gastrocolic ligament area were observed. Upon diagnosis, tumour markers CEA (144 ng/ml), CA 19-9 (558 U/ml), and lactate dehydrogenase (975 U/l) were elevated, and a slight iron-deficiency anaemia was detected. The patient had a KRAS mutation in codon 12 (VAL12 or G12V). Following evaluation of the case by the Multidisciplinary Committee, chemotherapy treatment was administered with XELOX-bevacizumab, and the patient received 6 cycles between May and August 2010. The response of the patient was verified biochemically and radiologically, with a PR at the hepatic level. Subsequent intervention was performed in October 2010, which included a left colectomy and right hepatectomy and resection of five left liver metastases. The pathological stage of the tumour was pT1N1M1, with total resection of the liver disease R0, and strong evidence of tumoural regression (Mandard TRG 3).

In January 2011, the patient had completed 6 months of systemic therapy, with 2 additional cycles of capecitabine and bevacizumab administered following surgery, but without oxaliplatin, due to neurotoxicity. The patient underwent periodic review of CEA and CT scans every 3-6 months, until April 2012, when two new liver metastases were observed in the follow-up CT scan. Positron emission tomography-CT was performed, which revealed questionable retroperitoneal adenopathy. Therefore, in May 2012, an exploratory laparotomy was performed, which confirmed the tumoural adenopathy with extension to the inferior vena cava vascular 

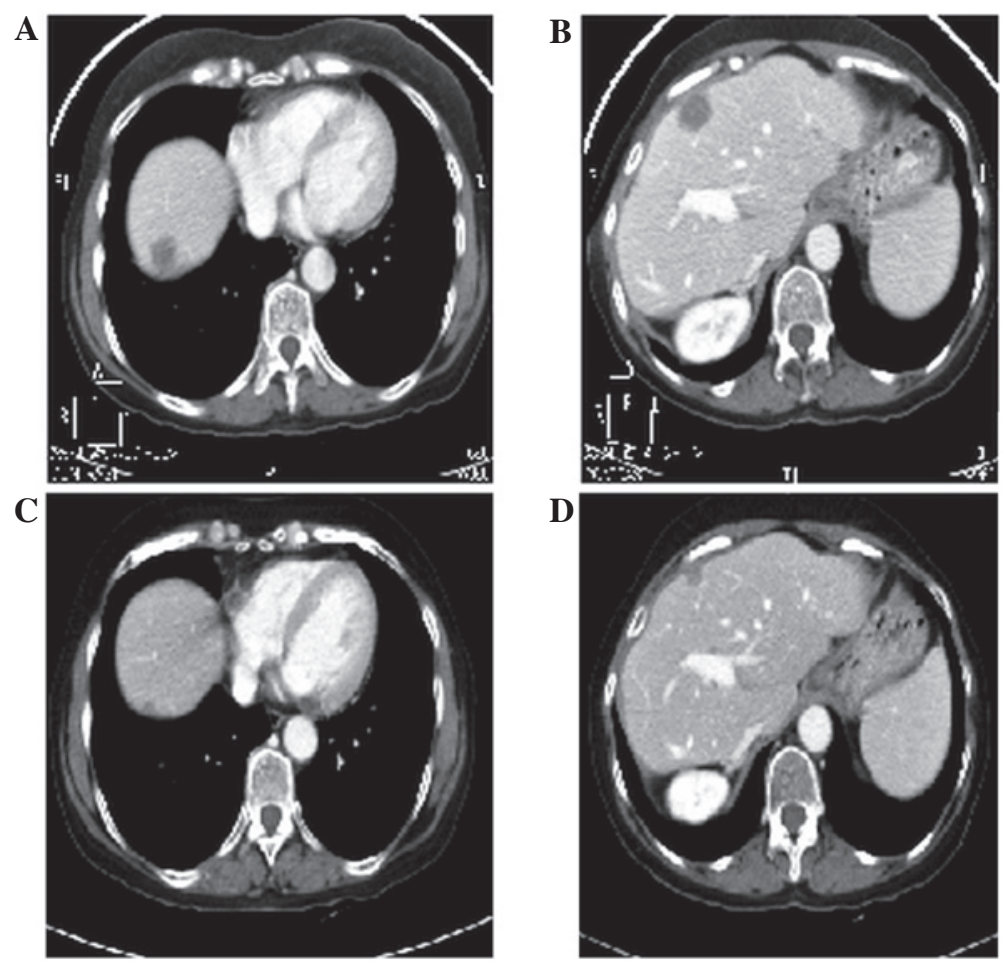

Figure 6. Case 4. Computed tomography scans at (A and B) baseline and (C and D) following 12 cycles of FOLFIRI (irinotecan, leucovorin and 5-fluorouracil)-aflibercept treatment, revealing partial radiological response at the hepatic level. (A) A 22-mm lesion in segment IV of the liver and (B) a $20 \mathrm{~mm}$ hepatic lesion in segment III were reduced (C and D, respectively) after 6 months of therapy.

wall and aorta. Consequently, due to the assessment of unresectable disease and a PS of 0 , FOLFIRI-based palliative chemotherapy was recommended, which was administered in June 2012. Aflibercept was administered from the third cycle following authorisation for use from the Compassionate Use Program. A CT scan performed following 7 cycles in October 2012, revealed a PR with a reduction in hepatic and ganglionic metastases. Subsequent to completion of 12 cycles, a CT scan in December 2012 revealed that the liver metastases were greatly reduced compared with the baseline CT scan (Fig. 6). Therefore, it was decided to continue with aflibercept monotherapy until progression.

With regard to toxicity, the patient had symptoms consistent with grade 1 acute neurotoxicity from irinotecan following completion of the first infusion, and grade 2 infusion reaction to aflibercept with lumbago in the fourth and fifth cycles, for which dexchlorpheniramine premedication was administered in later cycles. Cycles 8 and 12 were delayed, due to grade 2 neutropenia and respiratory infection. With the latter, the patient also had grade 2 mucositis and hypertension. During the maintenance period with aflibercept, the patient presented with hypertension, which reached grade 3 , but improved with adjustments to medication, as did grade 2 pharyngotonsillitis without neutropenia.

In April 2013, radiological progression was detected in the liver. The patient had persistent residual neurotoxicity from oxaliplatin. Subsequently, the patient was included in the MUTEX clinical trial at Virgen del Rocio Hospital (Seville, Spain), where the patient received cetuximab treatment until August 2013. During this time, the patient reported jaundice secondary to novel hepatic disease progression, which improved following placement of biliary endoprosthesis. In September 2013, the patient began 75\% FOLFOX.
Concurrently, multiple vertebral fractures were discovered with magnetic resonance imaging at the dorsolumbar level, due to osteoporosis, causing significant pain and requiring the use of a clamshell body jacket. Chemotherapy was suspended following 1 cycle, due to toxicity and deterioration of the patient's ECOG PS. The patient continued with supportive treatment until she succumbed to the disease in October 2013. The patient had reached an overall survival time (OS) of 41 months since diagnosis, and survival from the beginning of second-line treatment with aflibercept was 17 months. Progression free survival (PFS) following second-line treatment was 11 months.

Case 5. A 60 year-old male with no notable medical history, but with a family history of colon, gastric, pulmonary and testicular cancer, presented with fatigue in September 2012, and subsequently postprandial discomfort, slight diarrhoea and hyporexia. Following the detection of anaemia during routine testing in November 2012, the patient was diagnosed at Costa del Sol Hospital with well-differentiated stage IV adenocarcinoma at the hepatic angle of the colon with hepatic tumour involvement, along with lymph node metastases, which was assessed as unresectable. The KRAS gene of the patient was mutated in codon 13 (13ASP or G13D). The patient began palliative chemotherapy with FOLFOX6m-bevacizumab in December 2012, receiving 12 cycles of chemotherapy until June 2013, when tumour progression was detected. A CT scan revealed a suspicious rib lesion, which was confirmed to be metastatic, while growth of a pulmonary nodule from the primary tumour and the intra-abdominal ganglionic metastases was also observed. As the patient had a PS of 1 and elevated tumour markers (CEA, $8.4 \mathrm{ng} / \mathrm{ml}$; CA 19-9, $2351.6 \mathrm{U} / \mathrm{ml}$ ), the patient began second-line chemotherapy with 
FOLFIRI-aflibercept, with a $80 \%$ reduction in 5FU dose, due to prior toxicity. Unfortunately, the patient presented with a complication involving the $5 \mathrm{FU}$ infuser, which was considered unrelated to aflibercept treatment, and removal of the portacath was required. For this reason, after receiving 5 cycles and presenting with disease stabilisation at biochemical and radiological levels, irinotecan monotherapy was administered. To date, the patient presented with grade 2 hypertension, hyporexia, nausea, and diarrhoea, as well as grade 3 fatigue, requiring a reduction of the FOLFIRI dosage to improve tolerance. In November 2013, following a total of 8 cycles of treatment, chemotherapy was suspended following suspicion of tumour progression, due to progressively elevated tumour markers (CEA, $26 \mathrm{ng} / \mathrm{ml}$; CA 19-9, 2,200 U/ml) and clinical deterioration, with asthenia, abdominal pain and worsening PS. The patient succumbed to the disease in December 2013. The patient had a survival time of 6 months following the administration of second-line treatment with aflibercept.

\section{Discussion}

In the present case series, patients 1 and 5 presented with mutated KRAS in codon 13, specifically G13D/13ASP mutation, with the poorest evolution in survival occurring in patient 5 . The other three patients presented with mutations in codon 12 . Several studies have investigated the prognostic and predictive significance of different KRAS mutations, the G13D/13Asp mutation in particular exhibits an especially poor prognosis for patients (16-19). De Roock et al (16) suggested that in patients treated with supportive care with a KRAS G13D mutation have a poorer OS time (median, 3.6 months) compared with patients with wild-type $K R A S$ or other KRAS mutations (median OS time, 5.0 and 4.7 months, respectively). Another study demonstrated that patients with KRAS G13D mutation, who were treated with chemotherapy alone, without anti-EGFR treatment, presented with poorer response rates compared with patients with other mutations (response rate, 22.0 vs. $43.2 \%$, respectively; odds ratio, 0.40; $\mathrm{P}=0.032$ ) (17). These findings support the $\mathrm{OS}$ time of 12 months reported in case 5 in the present case series, which was lower than the OS time reported for the other present cases, and lower than the median OS time for patients with mCRC treated with similar therapy regimens (9).

The choice of first-line treatment in all cases in the present study was FOLFOX or XELOX in combination with bevacizumab (9). Until progression of disease is observed, possible treatment plans are FOLFIRI (20), FOLFIRI-bevacizumab (21) or FOLFIRI-aflibercept (13). The present study chose the latter based on the VELOUR study results (13). VELOUR was a multi-centre, randomised, placebo-controlled phase III trial, which aimed to compare the efficacy of aflibercept vs. placebo in combination with FOLFIRI as a second-line treatment for patients with $\mathrm{mCRC}$, who were previously treated with oxaliplatin. In the VELOUR study, patients with mCRC were randomised to receive FOLFIRI and aflibercept at $4 \mathrm{mg} / \mathrm{kg}$ intravenously every 2 weeks ( $\mathrm{n}=614$ ) vs. FOLFIRI and placebo $(\mathrm{n}=612)$, with stratification by PS and prior bevacizumab received. The aflibercept group demonstrated a superior median OS time [13.50 vs. 12.06 months; hazards ratio (HR) 0.817; $\mathrm{P}=0.0032$ ], PFS (6.90 vs. 4.67 months; HR 0.758; $\mathrm{P}<0.0001)$ and overall response rate $(19.8$ vs. $11.1 \% ; \mathrm{P}=0.0001)$ compared with the placebo group (13). In the subgroup analysis subsequently published, aflibercept was confirmed to be effective in all specified subgroups, even for patients that had been treated with first-line bevacizumab $(\sim 30 \%)$, with a median OS time of 12.5 vs. 11.7 months and PFS of 6.7 vs. 3.9 months for the experimental and control arm, respectively (20). This is consistent with results from other studies, which support the use of a continued antiangiogenic strategy for the treatment of mCRC (22-24).

The question remains whether aflibercept may offer an advantage over bevacizumab maintenance therapy, if the patient's disease has progressed following first-line treatment that already included bevacizumab. To date, no randomised study has answered that question. However, there is available data from pre-clinical studies that have made a comparative assessment of the activity of the two drugs. A recent study with mCRC tumour tissue models extracted from patients and xenografted mice (PDX cancer models) revealed differences in tumour growth control between the antiangiogenic treatments bevacizumab and aflibercept; the latter was revealed to be more effective in preventing tumour growth (25). Chiron et al (26) verified this data, also using PDX models, and revealed that administrating aflibercept during cancer progression following bevacizumab treatment facilitates maintenance of tumour growth inhibition, thus achieving a greater response compared with bevacizumab maintenance following tumour progression (26). This superior activity may be explained by the effect aflibercept has on VEGF and PIGF, since it better neutralises the resistance mechanisms that are activated by the tumour compared with bevacizumab $(27,28)$. In addition, it has been suggested that PIGF presence in patient serum may aid in the prediction of the optimal time for switching to antiangiogenic therapy prior to tumour progression taking place (25).

In the present case series, the combination of FOLFIRI-aflibercept was revealed to be effective in patients with mutated KRAS, who were treated previously with a combination of oxaliplatin and bevacizumab. The majority of patients received a greater number of aflibercept cycles than the median reported in the VELOUR study (present study, 12 cycles of FOLFIRI-aflibercept; VELOUR study, 7 cycles of aflibercept) (13), with manageable and reversible toxicity. In the present study, a partial radiological response was recorded in one patient and one CR was obtained in another patient. In addition, in the other present cases, the optimal morphological response observed in the CT scan image was able to predict an improved pathological response, which is known to be associated with prolonged survival (29). Furthermore, the majority of the present patients obtained a PFS greater than the median reported in the VELOUR study and in a subgroup of patients previously treated with bevacizumab (21). In addition, a median survival time of $\sim 47$ months from the initial treatment of the disease was reached, which is remarkable for a series entirely consisting of KRAS-mutated cases.

To the best of our knowledge, the present study is the first study to publish data from mCRC patients treated with aflibercept in routine clinical practice, outside of the clinical trial environment. The present study serves to contrast efficacy and safety results obtained from the pivotal VELOUR trial, and confirms that aflibercept is active and well-tolerated following bevacizumab treatment. 


\section{Acknowledgements}

Medical writing assistance, supported financially by Sanofi Spain (Barcelona, Spain), was provided by Mrs. A. Del Campo (Pivotal S.L., Madrid, Spain).

\section{References}

1. Jemal A, Bray F, Center MM, Ferlay J, Ward E and Forman D: Global cancer statistics. CA Cancer J Clin 61: 69-90, 2011.

2. Schmoll HJ, Van Cutsem E, Stein A, Valentini V, Glimelius B, Haustermans K, Nordlinger B, van de Velde CJ, Balmana J, Regula J, et al: ESMO Consensus Guidelines for management of patients with colon and rectal cancer. A personalized approach to clinical decision making. Ann Oncol 23: 2479-2516, 2012.

3. Heinemann V, Douillard JY, Ducreux M and Peeters M: Targeted therapy in metastatic colorectal cancer - An example of personalised medicine in action. Cancer Treat Rev 39: 592-601, 2013.

4. Casado-SaenzE,Feliu J,Gomez-España MA, Sanchez-Gastaldo A and Garcia-Carbonero R; SEOM: SEOM clinical guidelines for the treatment of advanced colorectal cancer 2013. Clin Transl Oncol 15: 996-1003, 2013.

5. Douillard JY, Oliner KS, Siena S, Tabernero J, Burkes R, Barugel M, Humblet Y, Bodoky G, Cunningham D, Jassem J, et al: Panitumumab-FOLFOX4 treatment and RAS mutations in colorectal cancer. N Engl J Med 369: 1023-1034, 2013.

6. Sorich MJ, Wiese MD, Rowland A, Kichenadasse G, McKinnon RA and Karapetis CS: Extended RAS mutations and anti-EGFR monoclonal antibody survival benefit in metastatic colorectal cancer: A meta-analysis of randomized controlled trials. Ann Oncol 26: 13-21, 2015.

7. Hassabo HM, Sahin IH and Kazmi SHA: Associations between patient (pt) colorectal cancer (CRC) tumour KRAS and BRAF mutation (mut) status and overall survival (OS). J Clin Oncol 32: 473,2014

8. Hurwitz H,Fehrenbacher L, Novotny W, Cartwright T, Hainsworth J, Heim W, Berlin J, Baron A, Griffing S, Holmgren E, et al: Bevacizumab plus irinotecan, fluorouracil, and leucovorin for metastatic colorectal cancer. N Engl J Med 350: 2335-2342, 2004.

9. Saltz LB, Clarke S, Díaz-Rubio E, Scheithauer W, Figer A, Wong R, Koski S, Lichinitser M, Yang TS, Rivera F, et al Bevacizumab in combination with oxaliplatin-based chemotherapy as first-line therapy in metastatic colorectal cancer: A randomised phase III study. J Clin Oncol 26: 2013-2019, 2008.

10. Kubicka S, Greil R, André T, Bennouna J, Sastre J, Van Cutsem E, von Moos R, Osterlund P, Reyes-Rivera I, Müller T, et al: Bevacizumab plus chemotherapy continued beyond first progression in patients with metastatic colorectal cancer previously treated with bevacizumab plus chemotherapy: ML18147 study KRAS subgroup findings. Ann Oncol 24: 2342-2349, 2013.

11. Holash J, Davis S, Papadopoulos N, Croll SD, Ho L, Russell M, Boland P, Leidich R, Hylton D, Burova E, et al: VEGF-Trap: A VEGF blocker with potent antitumour effects. Proc Natl Acad Sci USA 99: 11393-11398, 2002.

12. Temraz S, Mukherji D, Alameddine R and Shamseddine A: Methods of overcoming treatment resistance in colorectal cancer. Crit Rev Oncol Hematol 89: 217-230, 2014.

13. Van Cutsem E, Tabernero J, Lakomy R, Prenen H, Prausová J, Macarulla T, Ruff P, van Hazel GA, Moiseyenko V, Ferry D, et al: Addition of aflibercept to fluorouracil, leucovorin, and irinotecan improves survival in a phase III randomised trial in patients with metastatic colorectal cancer previously treated with an oxaliplatin-based regimen. J Clin Oncol 30: 3499-3506, 2012.

14. European Medicines Agency: Zaltrap Technical datasheet. http://www.ema.europa.eu/docs/es ES/document library/ EPAR_-_Product_Information/human/002532/WC500139484. pdf (last accessed 21st July, 2016).

15. Eisenhauer EA, Therasse P, Bogaerts J, Schwartz LH, Sargent D, Ford R, Dancey J, Arbuck S, Gwyther S, Mooney M, et al: New response evaluation criteria in solid tumours: Revised RECIST guideline (version 1.1). Eur J Cancer 45: 228-247, 2009.
16. De Roock W, Jonker DJ, Di Nicolantonio F, Sartore-Bianchi A Tu D, Siena S, Lamba S, Arena S, Frattini M, Piessevaux H, et al: Association of KRAS p.G13D mutation with outcome in patients with chemotherapy-refractory metastatic colorectal cancer treated with cetuximab. JAMA 304: 1812-1820, 2010.

17. Tejpar S, Celik I, Schlichting M, Sartorius U, Bokemeyer C and Van Cutsem E: Association of KRAS G13D tumour mutations with outcome in patients with metastatic colorectal cancer treated with first-line chemotherapy with or without cetuximab. J Clin Oncol 30: 3570-3577, 2012.

18. Andreyev HJ, Norman AR, Cunningham D, Oates JR and Clarke PA: Kirsten ras mutations in patients with colorectal cancer: the multicenter "RASCAL" study. J Natl Cancer Inst 90: 675-684, 1998

19. Pérez-Ruiz E, Rueda A, Pereda T, Alcaide J, Bautista D, Rivas-Ruiz F, Villatoro R, Pérez D and Redondo M. Involvement of K-RAS mutations and amino acid substitutions in the survival of metastatic colorectal cancer patients. Tumour Biol 33: $1829-1835,2012$

20. Tournigand C, André T, Achille E, Lledo G, Flesh M, Mery-Mignard D, Quinaux E, Couteau C, Buyse M, Ganem G, et al: FOLFIRI followed by FOLFOX6 or the reverse sequence in advanced colorectal cancer: A randomised GERCOR study. J Clin Oncol 22: 229-237, 2004

21. Tabernero J, Van Cutsem E, Lakomý R, Prausová J, Ruff P, van Hazel GA, Moiseyenko VM, Ferry DR, McKendrick JJ, Soussan-Lazard K, et al: Aflibercept versus placebo in combination with fluorouracil, leucovorin and irinotecan in the treatment of previously treated metastatic colorectal cancer: Prespecified subgroup analyses from the VELOUR trial. Eur J Cancer 50: 320-331, 2014.

22. Bennouna J, Sastre J, Arnold D, Österlund P, Greil R, Van Cutsem E, von Moos R, Viéitez JM, Bouché O, Borg C, et al: Continuation of bevacizumab after first progression in metastatic colorectal cancer (ML18147): A randomised phase 3 trial. Lancet Oncol 14: 29-37, 2013.

23. Grothey A, Sugrue MM, Purdie DM, Dong W, Sargent D, Hedrick E and Kozloff M: Bevacizumab beyond first progression is associated with prolonged overall survival in metastatic colorectal cancer: Results from a large observational cohort study (BRiTE). J Clin Oncol 26: 5326-5334, 2008.

24. Simkens LH, van Tinteren H, May A, ten Tije AJ, Creemers GJ, Loosveld OJ, de Jongh FE, Erdkamp FL, Erjavec Z, van der Torren AM, et al: Maintenance treatment with capecitabine and bevacizumab in metastatic colorectal cancer (CAIRO3): A phase 3 randomised controlled trial of the Dutch Colorectal Cancer Group. Lancet 385:1843-1852, 2015.

25. Chiron M, Bagley RG, Pollard J, Mankoo PK, Henry C, Vincent L, Geslin C, Baltes N and Bergstrom DA: Differential antitumour activity of aflibercept and bevacizumab in patient-derived xenograft models of colorectal cancer. Mol Cancer Ther 13: 1636-1644, 2014.

26. Chiron M, Bagley RG and Pollard J, Henry C, Mankoo P, Vincent L, Geslin C, Kloss T and Bergstrom DA: Switching to aflibercept treatment resulted in greater tumour responses than continuous bevacizumab treatment in patient-derived xenograft models of colorectal cancer (abstract). In: Proceedings of the AACR-NCI-EORTC International Conference, Boston, MA. Mol Cancer Ther 12 (11 Suppl): B2, 2013.

27. Cao Y: Positive and negative modulation of angiogenesis by VEGFR1 ligands. Sci Signal 2: re1, 2009.

28. Kopetz S, Hoff PM, Morris JS, Wolff RA, Eng C, Glover KY, Adinin R, Overman MJ, Valero V, Wen S, et al: Phase II trial of infusional fluorouracil, irinotecan, and bevacizumab for metastatic colorectal cancer: Efficacy and circulating angiogenic biomarkers associated with therapeutic resistance. J Clin Oncol 28: 453-459, 2010.

29. Shindoh J, Loyer EM, Kopetz S, Boonsirikamchai P, Maru DM, Chun YS, Zimmitti G, Curley SA, Charnsangavej C, Aloia TA and Vauthey JN: Optimal morphologic response to preoperative chemotherapy: An alternate outcome end point before resection of hepatic colorectal metastases. J Clin Oncol 30: 4566-4572, 2012. 